\title{
Unconventional motional narrowing in the optical spectrum of a semiconductor quantum dot
}

\author{
A. Berthelot ${ }^{1}$, I. Favero ${ }^{1}$, G. Cassabois ${ }^{1, *}$, C. Voisin $^{1}$, C. Delalande ${ }^{1}$, Ph. Roussignol $^{1}$, R. Ferreira $^{1}$ and J. M. Gérard ${ }^{2}$ \\ ${ }^{1}$ Laboratoire Pierre Aigrain, Ecole Normale Supérieure, 24 rue Lhomond 75231 Paris Cedex 5, France \\ ${ }^{2}$ CEA-CNRS-UJF "Nanophysics and Semiconductors" Laboratory, \\ CEA/DRFMC/SP2M, 17 rue des Martyrs 38054 Grenoble Cedex 9, France
}

(Dated: July 16, 2018)

\begin{abstract}
Motional narrowing refers to the striking phenomenon where the resonance line of a system coupled to a reservoir becomes narrower when increasing the reservoir fluctuation. A textbook example is found in nuclear magnetic resonance, where the fluctuating local magnetic fields created by randomly oriented nuclear spins are averaged when the motion of the nuclei is thermally activated. The existence of a motional narrowing effect in the optical response of semiconductor quantum dots remains so far unexplored. This effect may be important in this instance since the decoherence dynamics is a central issue for the implementation of quantum information processing based on quantum dots. Here we report on the experimental evidence of motional narrowing in the optical spectrum of a semiconductor quantum dot broadened by the spectral diffusion phenomenon. Surprisingly, motional narrowing is achieved when decreasing incident power or temperature, in contrast with the standard phenomenology observed for nuclear magnetic resonance.
\end{abstract}

PACS numbers: 78.67.Hc, 78.55.Cr, 05.40.-a

In the seminal work on motional narrowing by Bloembergen et al., relaxation effects in nuclear magnetic resonance were beautifully explained by taking into account the influence of the thermal motion of the magnetic nuclei upon the spin-spin interaction [1]. The general treatment of relaxation processes for a system interacting with a reservoir was later formulated by Kubo in a stochastic theory that assumes random perturbations of the system by a fluctuating environment [2]. Depending on the relative magnitude of the spectral modulation amplitude and the inverse of the modulation correlation time, the relaxation dynamics is either in the slow modulation limit, where the optical line-shape reflects directly the statistical distribution of the different system energies, or in the fast modulation limit where the fluctuation is smoothed out and the line-shape is motionally narrowed into a Lorentzian profile. The relevance of motional narrowing for the description of relaxation phenomena has spread throughout many different fields, such as spin relaxation in semiconductors [3] , vibrational dephasing in molecular physics [4], or phase noise in optical pumping [5].

The optical spectrum of a material system with localized, zero-dimensional electronic states provides a generic example of the influence of a fluctuating environment on the coherence relaxation dynamics. In that case, the perturbing interactions induce a stochastic shift over time of the optical spectrum, resulting in the so-called spectral diffusion effect, which was observed for rare-earth ions $\underline{6}$, molecules [7], or semiconductor quantum dots [8, 9]. In this latter system, impurities, defects or localized charges in the vicinity of a quantum dot induce micro-electric fields that shift the quantum dot emission line through the quantum confined Stark effect. The fluctuation of the quantum dot environment thus randomize the emission energy over a spectral range $\Sigma$ on a characteristic time scale $\tau_{c}$. Spectral diffusion under light illumination was reported with jitters of the quantum dot emission energy from hundreds $\mu \mathrm{eV}$ to few meV on time scales ranging from milliseconds to minutes $[8,9,10]$. However, to the best of our knowledge, no evidence for motional narrowing $\left(\Sigma \tau_{c} \ll \hbar\right)$ was pointed out for the spectral diffusion phenomenon in quantum dots.

We present here the experimental evidence for motional narrowing in the optical spectrum of a semiconductor quantum dot. High-resolution Fourier-transform spectroscopy performed on the photoluminescence signal of a single InAs/GaAs quantum dot allows the determination of both width and shape of the emission line. A crossover from Lorentzian to Gaussian profiles of the zero-phonon line is observed while its linewidth increases. We obtain a quantitative agreement with Monte Carlo simulations where the fluctuating environment is modelled by the random capture and escape of carriers in traps located in the quantum dot vicinity. We show that motional narrowing is achieved when decreasing the temperature or the incident power because of the asymmetry of the capture and escape mechanisms. Our study provides a counterintuitive example of motional narrowing compared to the standard phenomenology described in nuclear magnetic resonance.

We study self-assembled InAs/GaAs quantum dots grown by molecular beam epitaxy in the StranskiKrastanow mode, and micro-photoluminescence measurements under non-resonant excitation are performed in the far field using the experimental setup described in Ref. [1]. In order to accurately characterize the quantum dot emission spectrum, the photoluminescence signal arising from a single quantum dot is analyzed by high-resolution Fourier-transform spectroscopy [11]. The Fourier-transform technique is implemented in the detection part of the setup where the photoluminescence 
signal passes through a Michelson interferometer placed in front of a $32 \mathrm{~cm}$ grating spectrometer. The signal is detected by a low noise Si-based photon counting module. With a translation stage, we vary the time $t$ for propagation in one arm of the interferometer and record interferograms of the photoluminescence emission $I(t)=I_{0}\left(1+C(t) \cos \left(E_{0} t / \hbar\right)\right)$, where $I_{0}$ is the average photoluminescence signal intensity, $E_{0}$ the central detection energy, and $C(t)$ the interference contrast. In the two cases of Lorentzian or Gaussian line-shapes, a Fouriertransform analysis shows that the interference contrast decay is exponential or Gaussian, respectively. As we will see below, the implementation of this technique in single quantum dot spectroscopy allows an accurate determination of both width and shape of the emission line. As far as the linewidth is concerned, sub- $\mu \mathrm{eV}$ measurements can be performed by using this interferometric correlation technique [1]. For the determination of the line-shape, our technique allows a high-resolution sampling of the Fourier-transform of the spectrum on typically thousands of points. This value is by two orders of magnitude larger than the average number of illuminated pixels in a charge-coupled device in the case of a multichannel detection in the spectral domain. The drawbacks of our Fourier-transform technique are the long acquisition times of roughly one hour per interferogram, and the required extremely high mechanical stability of the system during the interferogram acquisition.
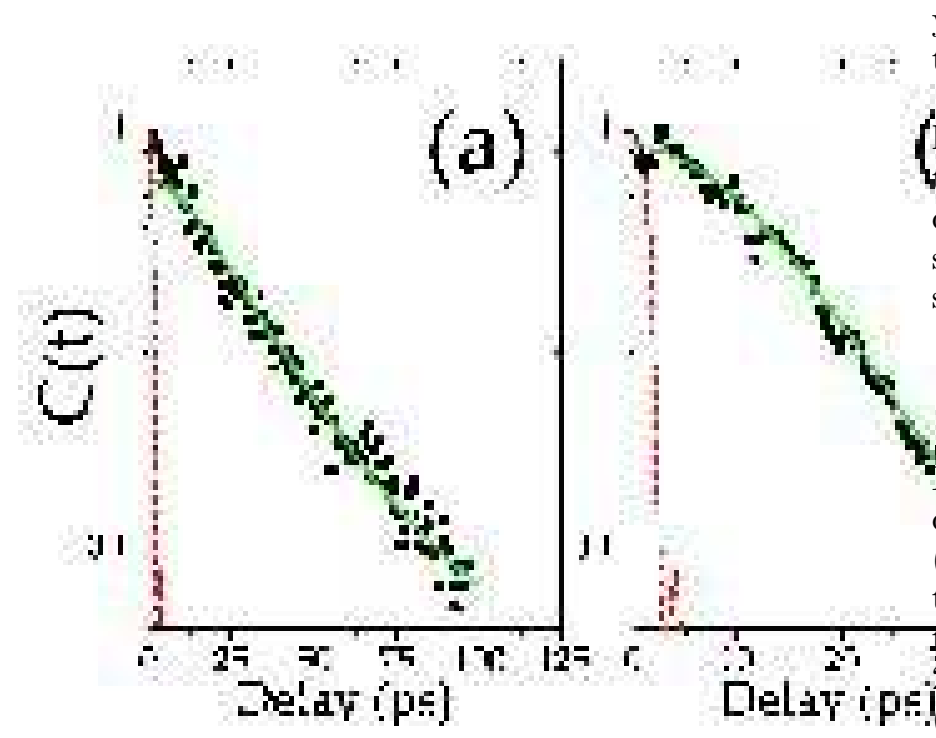

FIG. 1: Interferogram contrast $C(t)$ of the photoluminescence signal of a single InAs/GaAs quantum dot at $10 \mathrm{~K}$, on semilogarithmic plots, for three different incident powers: 0.18 (a), 0.72 (b), and $2.88 \mathrm{~kW} \cdot \mathrm{cm}^{-2}$ (c). Data (squares), system response function (dotted line, in red), theoretical fits (solid line, in green) obtained by the convolution of the system response function with Eq. (1), with $\Sigma \tau_{c} / \hbar \sim 0.6$ (a), 1.05 (b), and $1.35(\mathrm{c})$, are plotted as a function of the delay $t$.
In Fig. 10 we display the measured (squares) interference contrast $C(t)$ for the emission spectrum of a single InAs/GaAs quantum dot at $10 \mathrm{~K}$, on semi-logarithmic plots, for three different incident powers: 0.18 (a), 0.72 (b), and $2.88 \mathrm{~kW} \cdot \mathrm{cm}^{-2}$ (c). We first observe that the coherence relaxation dynamics becomes faster when increasing the incident power. Moreover, we notice a gradual modification in the shape of $C(t)$. At low power (Fig. 1(a)), the interference contrast decay is quasiexponential with a time constant of 29 ps, thus corresponding to a quasi-Lorentzian profile with a full width at half maximum (FWHM) of $45 \mu \mathrm{eV}$. At higher power (Fig. 1(b)), $C(t)$ has a Gaussian decay at short times $(t \leq 15 \mathrm{ps})$, and remains exponential at longer times. The line has an intermediate profile, and here its FWHM is $105 \mu \mathrm{eV}$. At the highest incident power (Fig. 1( (c)), the interference contrast decay is predominantly Gaussian, thus corresponding to a quasi-Gaussian profile, with a FWHM of $155 \mu \mathrm{eV}$.

This crossover from a Lorentzian to a Gaussian lineshape when increasing the incident power reveals an original relaxation dynamics in semiconductor physics. In fact, in bulk semiconductors or in quantum wells, the standard phenomenology is the exact opposite. An increasing number of photo-created carriers leads to the activation of carrier-carrier Coulomb correlations, and induces the inverse transition from an inhomogeneously broadened Gaussian line to a homogeneous Lorentzian one 12. In the following, we provide a quantitative analysis of our measurements in the framework of the Kubo theory.

Appl fing the stochastic theory of line-shape and re-

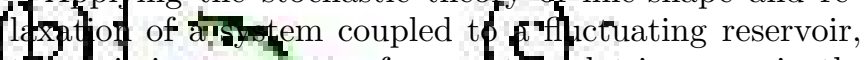
ithe emi ssion spectium of a quahtund döt is conveniently charact rized in theteme domain and, assuming Gaussian fluctuation, the Fqurier-transform of the intensity spectru $n$ has the generatexpression [2]:

$$
\left.C(t)=\exp _{-2}\left[-\frac{\Sigma^{2} \tau_{c}^{2}}{\hbar^{2}}\left(\operatorname{ext}-\frac{t}{\tau_{c}}\right)+\frac{t}{\tau_{c}}-1\right)\right]
$$

In the limit of slow modulation $\left(\Sigma \tau_{c} \gg \hbar\right)$, the charact ristic function $C(t)$ has a Gaussian decay $\left(C(t) \sim \operatorname{Xp}\left(-\Sigma_{1}^{2} t^{2} / 2 \hbar^{2}\right)\right)$ : the spetyrum, reflects directly the sta istical distribution of the emission energies, and the "ling shape is Gallssian in a FWHM given by $2 \sqrt{2 \ln 2} 2$. In the opposite limit of fast modulation

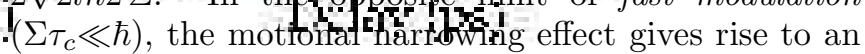
exponential decoherence $\left(C(t) \sim \exp \left(-\Sigma^{2} \tau_{c} t / \hbar^{2}\right)\right)$ with a decay time $T_{2}=\hbar^{2} / \Sigma^{2} \tau_{c}$ : the fluctuation is smoothed out by the fast modifications of the reservoir configuration, and the line-shape becomes Lorentzian with a FWHM given by $2 \Sigma^{2} \tau_{c} / \hbar$. Indeed, when motional narrowing occurs, the linewidth is reduced by a factor of the order of $\Sigma \tau_{c} / \hbar$.

A comparison between our experimental data and the general expression of $C(t)$ given above allows us to characterize the transition from the exponential to the Gaussian relaxation observed in Fig. [1 For this purpose, we 
convolute Eq. (1) with the system response function (dotted lines in Fig. (1) which is obtained under white light illumination. The calculated fits are displayed in solid line in Fig. 10 We obtain an excellent agreement with increasing values of $\Sigma \tau_{c} / \hbar$ of 0.6 in (a), 1.05 in (b), and 1.35 in (c), where the correlation time $\tau_{c}$ has a constant value of $10 \mathrm{ps}$. The gradual increase of $\Sigma \tau_{c} / \hbar$ demonstrates the transition from the fast modulation limit to the slow modulation one when increasing the incident power. As a matter of fact, it shows that motional narrowing occurs for low excitation. At first glance, one may think that increasing the number of photo-created carriers in the structure should induce some additional motion in the environment, so that the quantum dot line should be motionally narrowed in the high excitation limit. It is in fact the exact opposite, and we explain below the origin of this unconventional phenomenology.

The existence of a fluctuating environment around the quantum dot originates from the presence of impurities, defects in the barrier material or in the wetting layer. Furthermore, this latter heterostructure is far from being an ideal two-dimensional quantum well so that the unavoidable interface roughness gives also rise to localized states in the quantum dot surroundings [9, 13, 14]. The characteristic parameters $\Sigma$ and $\tau_{c}$ are thus determined by the population fluctuation in the various traps located around the quantum dot. We present Monte Carlo simulations of the carrier dynamics in the reservoir for the understanding of the motional narrowing effect in quantum dots.

In our model, the fluctuating reservoir consists in $N$ uncorrelated traps which individually induce a Stark shift $\Delta$ of the quantum dot line. This simple scheme has been chosen for the sake of clarity. More realistic geometries can be used but do not alter the conclusions. Each trap is loaded with a characteristic capture time $\tau_{\downarrow}$, and emptied with an escape time $\tau_{\uparrow}$ (Fig. 2(a)). In our timedependent simulations, the random modifications of the traps population result in fluctuation over time $\delta E(t)$ of the transition energy around a mean value $E_{0}$. Then, the spectral modulation amplitude $\Sigma$ and the correlation time $\tau_{c}$ are calculated by computing the correlation function of the energy fluctuation $X(t)$, which is defined by the configuration average $X(t)=\langle\delta E(t) \delta E(0)\rangle$ 2]. This correlation function is expected to have an exponential decay given by $X(t)=\Sigma^{2} \exp \left(-t / \tau_{c}\right)$ [2]. In Fig. 2]b), we display the correlation function $X(t)$ computed for $N=50$ and averaged over one thousand configurations. We observe on this semi-logarithmic plot the exponential decay of $X(t)$, from which we extract $\Sigma$ and $\tau_{c}$. In Fig. 2(c) we display the correlation time $\tau_{c}$ normalized to the capture time $\tau_{\downarrow}$ as a function of the ratio $\tau_{\downarrow} / \tau_{\uparrow}$. We observe that our calculations are fitted by using the simple expression:

$$
\frac{1}{\tau_{c}}=\frac{1}{\tau_{\uparrow}}+\frac{1}{\tau_{\downarrow}}
$$

which shows that the correlation time is inversely proportional to the total fluctuation rate of the traps population.

Finally, in Fig. 2(d), we display the spectral modulation amplitude $\Sigma$ normalized to its saturation value $\Sigma_{s}$. Our numerical simulations are well reproduced by using the expression:

$$
\Sigma=\frac{\sqrt{N} \Delta}{\sqrt{\frac{\tau_{\uparrow}}{\tau_{\downarrow}}}+\sqrt{\frac{\tau_{\downarrow}}{\tau_{\uparrow}}}}
$$

which can also be analytically derived by assuming ergodicity of the reservoir. The saturation value $\Sigma_{s}$ of the spectral modulation amplitude is thus given by $\sqrt{N} \Delta / 2$. In semiconductor nanostructures, at low temperature carrier escape is known to be much less efficient than the capture process [12]. When $\tau_{\downarrow} / \tau_{\uparrow} \ll 1$, the correlation time $\tau_{c}$ has approximately a constant value given by $\tau_{\downarrow}$ whereas $\Sigma$ scales as $2 \Sigma_{s} \sqrt{\tau_{\downarrow} / \tau_{\uparrow}}$. Therefore, an activation of the escape process by raising the incident power will lead to an increase of $\Sigma \tau_{c} / \hbar$, in qualitative agreement with the phenomenology discussed above.

On the basis of our simple microscopic model, we present a quantitative interpretation of our experiments at $10 \mathrm{~K}$ as a function of incident power. In Fig. [3 we display the measured values of the FWHM (squares) and $\Sigma \tau_{c} / \hbar$ (circles, inset) as a function of the excitation density, on a semi-logarithmic scale. These two parameters characterize the width of the line-profile and its shape since the crossover from the exponential to the Gaussian decoherence dynamics occurs around $\Sigma \tau_{c} / \hbar \sim 1$. We confront our data with the theoretical values (solid lines) of the FWHM and $\Sigma \tau_{c} / \hbar$ that are calculated with Eq. (13), and we observe a fair agreement for the set of parameters $\Sigma_{s} \sim 400 \mu \mathrm{eV}, \tau_{\downarrow} \sim 10 \mathrm{ps}$, and $1 / \tau_{\uparrow}=\left(1 / \tau_{0}\right) \sqrt{P}$ where $\tau_{0} \sim 1.6 \mathrm{~ns}$ and $P$ is in unit of $1 \mathrm{~kW} . \mathrm{cm}^{-2}$.

The asymmetry between the power dependences of $\tau_{\downarrow}$ and $\tau_{\uparrow}$ stems from the existence of different microscopic processes of various efficiencies in semiconductor physics 12. The constant value of $10 \mathrm{ps}$ for $\tau_{\downarrow}$ is consistent with an optical-phonon assisted capture in the traps around the quantum dot [15]. On the other hand, the inverse process of carrier escape by optical-phonon absorption is strongly inhibited at low temperature, so that it does not significantly contribute to the value of $\tau_{\uparrow}$. In fact, the escape rate dependence on incident power is characteristic of Auger-type processes. In the elastic collision of two carriers where one is ejected from the trap while a delocalized carrier relaxes in energy, the escape rate is proportional to the density of delocalized carriers, which increases with incident power. In the expression $1 / \tau_{\uparrow}=\left(1 / \tau_{0}\right) \sqrt{P}$, the sublinear increase of the escape rate with $P$ can come from the fact that the actual density of delocalized carriers is governed by bimolecular interband radiative recombination [16] or Auger scattering [17], which both result in a square root dependence 
of the carrier density on $P$. Lastly, we note that in the limit of low incident power, the saturation value $\Sigma_{s}$ given by $\sqrt{N} \Delta / 2$ may be power-dependent due to the reduced number of active traps that are populated from the reservoir of delocalized carriers. Systematic measurements as a function of temperature and incident power on several quantum dots (to be described elsewhere) allow us to discriminate between the variations of $N$ and $\tau_{\uparrow}$ in our experiments. The power-dependence of $N$ is found to be negligible in the investigated range of incident power, in agreement with the assumptions made for our calculations.

Temperature-dependent experiments performed at low incident power $\left(0.07 \mathrm{~kW} . \mathrm{cm}^{-2}\right)$ reveal the same striking phenomenology, namely we observe a gradual increase of $\Sigma \tau_{c} / \hbar$ with temperature which demonstrates that motional narrowing occurs at low temperature. However, the analysis of the data recorded as a function of temperature requires a more careful procedure because the line-profile exhibits broad side-bands around the central so-called zero-phonon line [18, 19]. These side-bands are related to the radiative recombination assisted by the emission and the absorption of acoustic phonons. At low temperature $(\mathrm{T}<25 \mathrm{~K})$, their contribution to the total integrated intensity of the photoluminescence signal is negligible and can be safely neglected in the data interpretation, as done for our first set of measurements as a function of incident power at $10 \mathrm{~K}$. Above $25 \mathrm{~K}$, the presence of broad lateral side-bands around the zero-phonon line results in an interference contrast $C(t)$ with an additional fast component, the decay of which follows our system response function (Fig. 4(a)). By adding a constant term to the line-profile extracted from Eq. (1), we are able to fit the whole decay dynamics of the interference contrast (solid line, in green in (Fig. 4(a)), and thus separate the relative contributions of the lateral side-bands and zerophonon line (line+empty circles, in blue in (Fig. 4(a)).

In Fig. 4(b) we display the measured values of the FWHM (squares) and $\Sigma \tau_{c} / \hbar$ (circles, inset) of the zerophonon line as a function of the temperature. In the temperature-dependent measurements, the crossover from a Lorentzian to a Gaussian shape of the zero-phonon line occurs when increasing the temperature. More precisely, a smooth transition analog to the one presented in Fig. 1 is recorded in the temperature range between $30 \mathrm{~K}$ and $45 \mathrm{~K}$. We then confront our data with the theoretical values (solid lines) of the FWHM and $\Sigma \tau_{c} / \hbar$ that are calculated with Eq. (13), by assuming that:

$$
\begin{aligned}
& \frac{1}{\tau_{\downarrow}}=\frac{1}{\tau_{1}}\left(1+n_{1}(T)\right)+\frac{1}{\tau_{2}}\left(1+n_{2}(T)\right) \\
& \frac{1}{\tau_{\uparrow}}=\frac{1}{\tau_{1}} n_{1}(T)+\frac{1}{\tau_{2}} n_{2}(T)+\frac{1}{\tau_{3}}
\end{aligned}
$$

In Eq. (5) the constant term inversely proportional to $\tau_{3}$ accounts for the contribution of Auger processes to carrier escape, as discussed above, and its magnitude is fixed by the incident power. In the interpretation of our temperature-dependent measurements, the relevant microscopic processes for the carrier dynamics in the traps are phonon-assisted mechanisms where the capture (escape) is thermally-activated due to the emission (absorption) of an acoustic phonon of mean energy $E_{1}$ or an optical phonon of energy $E_{2}$. The capture and escape efficiencies are proportional to $\left(1+n_{i}(t)\right)$ and $n_{i}(t)$ respectively, where $n_{i}(t)$ is a Bose-Einstein occupation factor given by $1 /\left(\exp \left(E_{i} / k T\right)-1\right)$. In Fig. 4l b), we observe a fair agreement between the data and the calculations for the set of parameters $\Sigma_{s} \sim 400 \mu \mathrm{eV}, \tau_{1} \sim 35 \mathrm{~ns}, \tau_{2} \sim 10 \mathrm{ps}$, $\tau_{3} \sim 6.5 \mathrm{~ns}, E_{1} \sim 1 \mathrm{meV}$, and $E_{2} \sim 20 \mathrm{meV}$. This latter activation energy is smaller than the characteristic optical phonon energy of $36 \mathrm{meV}$ in bulk GaAs, and this difference may come from strain and confinements effects in the traps formed in the GaAs barrier or in the InAs wetting layer 20]. As far as the carrier dynamics in the traps is concerned, we find that in our experiments performed below $50 \mathrm{~K}$, the capture process is dominated by optical phonon emission $\left(\tau_{\downarrow} \sim \tau_{2}\right)$. On the other hand, the thermal activation of the escape one is predominantly due to acoustic phonon absorption below $40 \mathrm{~K}$, with an onset of optical phonon absorption around 40K. Finally, we conclude that in the investigated range of temperature, we are always in the regime where the capture process is much more efficient than the escape one $\left(\tau_{\downarrow} / \tau_{\uparrow} \ll 1\right)$, in exact similarity to the power-dependent experiments at $10 \mathrm{~K}$.

The asymmetry of the capture and escape mechanisms is in fact the fundamental reason why motional narrowing strikingly occurs when decreasing the incident power or the temperature. If both processes had the same efficiency $\left(\tau_{\uparrow} \sim \tau_{\downarrow}\right)$, we would have $\tau_{c} \sim \tau_{\downarrow} / 2$ and $\Sigma \sim \Sigma_{s}$ which means that the spectral modulation amplitude would not depend on the time constant $\tau_{\downarrow}$. Therefore, the ratio $\Sigma \tau_{c} / \hbar$ could only decrease when increasing the reservoir excitation. This situation corresponds to the well-known phenomenology from nuclear magnetic resonance where the activation of the nuclei motion induces the motional narrowing effect. In the present case where $\tau_{\downarrow} / \tau_{\uparrow} \leq 10^{-2}$, we are in the opposite regime where the correlation time is merely constant with relative variation smaller than $10^{-2}$ whereas the spectral modulation amplitude shows a steep increase with $\tau_{\downarrow} / \tau_{\uparrow}\left(\Sigma \propto \sqrt{\tau_{\downarrow} / \tau_{\uparrow}}\right)$. The ratio $\Sigma \tau_{c} / \hbar$ thus increases when increasing the reservoir excitation.

We conclude that motional narrowing in the optical spectrum of a quantum dot broadened by spectral diffusion occurs in the unexpected regime of low temperature and low incident power. This result is of great importance for the applications of quantum dots to quantum information processing. In fact, our study demonstrates that the decoherence dynamics can still be characterized by a decoherence time $T_{2}$ even in the presence of fluctuating local electric fields causing spectral diffusion. In that case, the zero-phonon line has a Lorentzian profile and its width is not given by the intrinsic radiative limit, 
but by the extrinsic reservoir fluctuation dynamics. In particular, we provide a straightforward interpretation to the widely debated issue of the origin of the temperature dependence of the zero-phonon linewidth. Eventually, we show that the motional narrowing effect strongly reduces the environment-induced dephasing since the zerophonon linewidth is approximately $\Sigma \tau_{c} / \hbar$ smaller than the spectral modulation amplitude $2 \sqrt{2 \ln 2} \Sigma$ due to spectral diffusion. This unconventional motional narrowing opens a novel route towards the control of environmentinduced dephasing for the applications of quantum dots to quantum information.

*Electronic address: Guillaume.Cassabois@lpa.ens.fr References
[1] Bloembergen, N., Purcell, E. M. \& Pound, R. V. Relaxation effects in nuclear magnetic resonance, Phys. Rev. 73, 679-715 (1948).

[2] Kubo, R. A stochastic theory of line-shape and relaxation, in Fluctuation, Relaxation and Resonance in Magnetic Systems (ed D. Ter Haar) 23-68 (Oliver and Boyd, Edinburgh, 1962).

[3] Dyakonov, M. I. \& Perel, V. I. Spin orientation of electrons associated with the interband absorption of light in semiconductors, Sov. Phys. JETP 33, 1053-1059 (1971).

[4] Oxtoby, D. W. Hydrodynamic theory of vibrational dephasing in liquids, J. Chem. Phys. 70, 2605-2610 (1979).

[5] Eberly, J. H., Wódkiewicz, K. \& Shore, B. W. Noise in strong laser-atom interactions: phase telegraph noise, Phys. Rev. A 30, 2381-2389 (1984).

[6] Flach, R., Hamilton, D. S., Selzer, P. M. \& Yen, W. M. Time-Resolved Fluorescence Line-Narrowing Studies in $\mathrm{LaF}_{3}: \mathrm{Pr}^{3+}$, Phys. Rev. Lett. 35, 1034-1037 (1975).

[7] Ambrose, W. P. \& Moerner, W. E. Fluorescence spectroscopy and spectral diffusion of single impurity molecules in a crystal, Nature 349, 225-227 (1991).

[8] Empedocles, S. A., Norris, D. J. \& Bawendi, M. G. Photoluminescence spectroscopy of single CdSe nanocrystallite quantum dots, Phys. Rev. Lett. 77, 3873-3876 (1996).

[9] Robinson, H. D. \& Goldberg, B. B. Light-induced spectral diffusion in single self-assembled quantum dots, Phys. Rev. B 61, R5086-5089 (2000).

[10] Besombes, L., Kheng, K., Marsal, L. \& Mariette, H. Fewparticle effects in single CdTe quantum dots, Phys. Rev. B 65, 121314-121317 (2002).

[11] Kammerer, C., Cassabois, G., Voisin, C., Perrin, M., Delalande, C., Roussignol, Ph. \& Gérard, J. M. Interferometric correlation spectroscopy in single quantum dots, Appl. Phys. Lett. 81, 2737-2739 (2002).

[12] J. Shah, in Ultrafast spectroscopy of semiconductors and semiconductor nanostrutures (Spinger-Verlag, Berlin, 1998).

[13] Gérard, J. M., Genin, J. B., Lefebvre, J., Moison, J. M., Lebouche, N. \& Barthe, F. Optical investigation of the self-organized growth of InAs/GaAs quantum boxes, J. Cryst. Growth 150, 351-356 (1995).

[14] Heitz, R., Ramachandran, T. R., Kalburge, A., Xie, Q.,
Mukhametzhanov, I., Chen, P. \& Madhukar, A. Observation of reentrant $2 \mathrm{D}$ to $3 \mathrm{D}$ morphology transition in highly strained epitaxy: InAs on GaAs, Phys. Rev. Lett. 78, 4071-4074 (1997).

[15] Lohner, A., Woerner, M., Elsaesser, M. \& Kaiser, W. Picosecond capture of photoexcited holes by shallow acceptors in p-type GaAs, Phys. Rev. Lett. 68, 3920-3923 (1992).

[16] J. E. Carroll, in Rate equation in semiconductor electronics (Cambridge University Press, 1985).

[17] O'Hara, K. E., Gullingsrud, J. R. \& Wolfe, J. P. Auger decay of excitons in $\mathrm{Cu}_{2} \mathrm{O}$, Phys. Rev. B 60, 10872-10885 (1999).

[18] Besombes, L., Kheng, K., Marsal, L. \& Mariette, H. Acoustic phonon broadening mechanism in single quantum dot emission, Phys. Rev. B 63, 155307-155311 (2001).

[19] Favero, I., Cassabois, G., Ferreira, R., Darson, D., Voisin, C., Tignon, J., Delalande, C., Bastard, G., Roussignol, Ph. \& Gérard, J. M. Acoustic phonon side-bands in the emission line of single InAs/GaAs quantum dots, Phys. Rev. B 68, 233301-233304 (2003).

[20] Jusserand, B. \& Cardona, M., in Light scattering in solids V (eds Cardona, M. \& Guntherrodt G.) 49-152 (Springer-Verlag, Heidelberg, 1989).

\section{Acknowledgements}

We thank C. Ciuti and B. Gayral for helpful discussions. LPA-ENS is "unité mixte (UMR 8551) de l'ENS, du CNRS, des Universités Paris 6 et 7". This work is financially supported by the region Ile de France through the project SESAME E-1751.

\section{Competing financial interest}

The authors declare that they have no competing financial interests. 


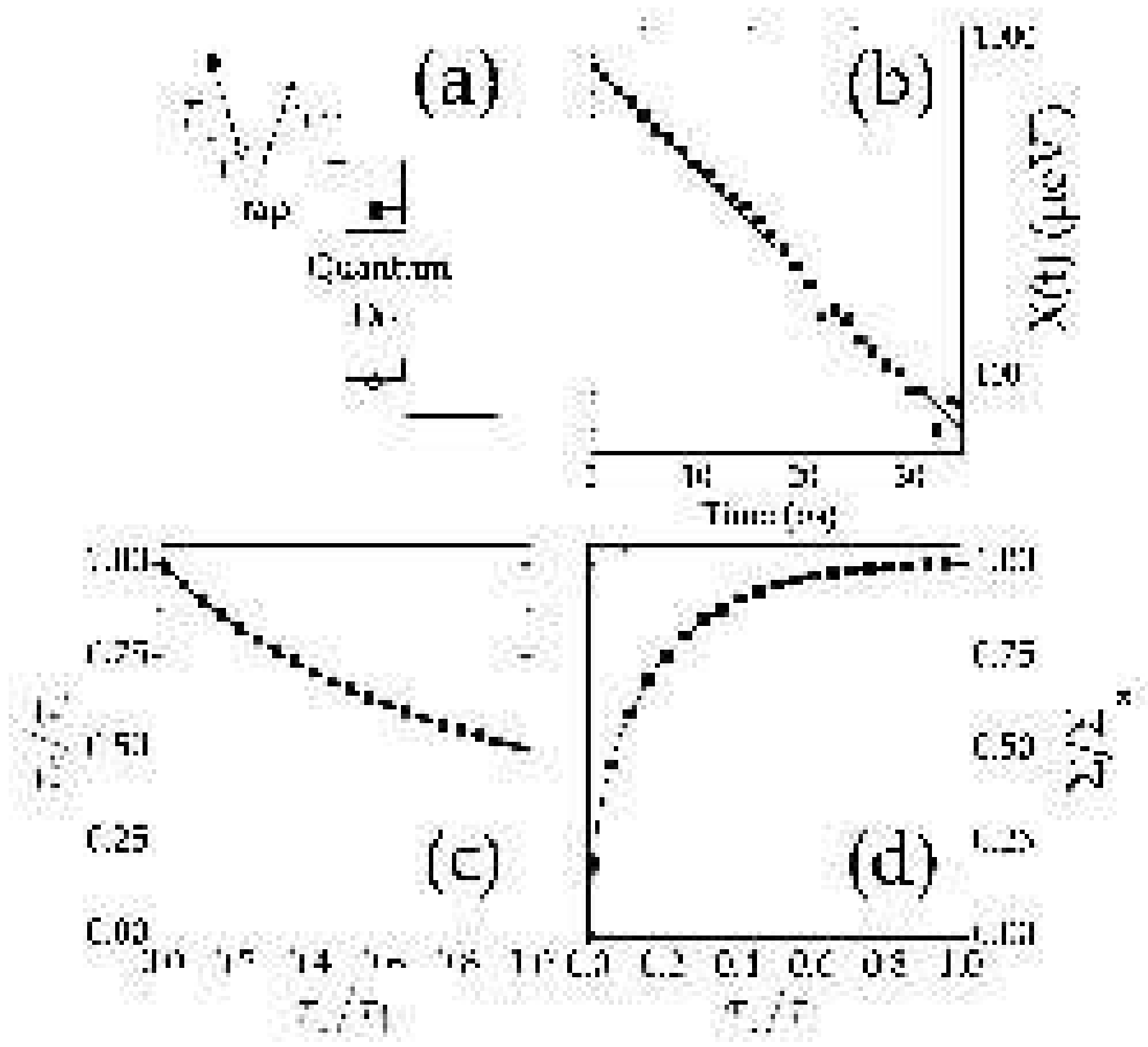

FIG. 2: (a) Schematic of the quantum dot and its fluctuating environment: $\tau_{\downarrow}$ and $\tau_{\uparrow}$ are the capture and escape times of carriers. (b) Correlation function of the energy fluctuation as a function of time, on a semi-logarithmic plot: Monte-Carlo simulations (squares) and exponential fit (solid line). (c) Correlation time $\tau_{c}$ normalized to the capture time $\tau_{\downarrow}$ versus $\tau_{\downarrow} / \tau_{\uparrow}$ and (d) spectral modulation amplitude $\Sigma$ normalized to its saturation value $\Sigma_{c}$ versus $\tau_{\downarrow} / \tau_{\uparrow}$ : Monte-Carlo simulations (squares), and fits (solid lines) according to Eq. (2) for (c) and Eq. (3) for (d). 


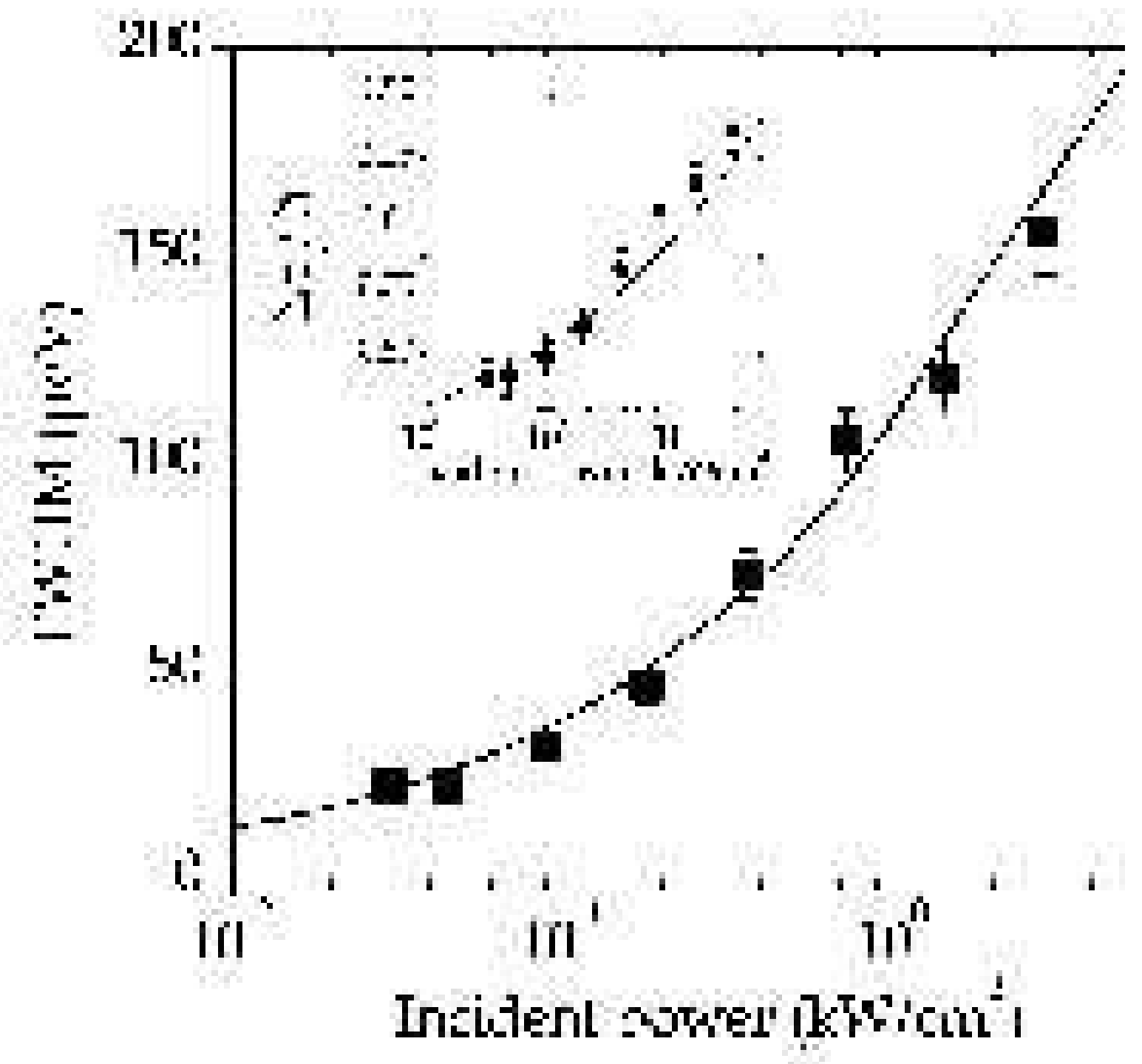

FIG. 3: Full width at half maximum (FWHM) and $\Sigma \tau_{c} / \hbar$ (inset) of the zero-phonon line of a single quantum dot, at 10K. Data (symbols), and calculations (solid lines) based on Eq. (13) are plotted as a function of incident power, on a semi-logarithmic scale. 

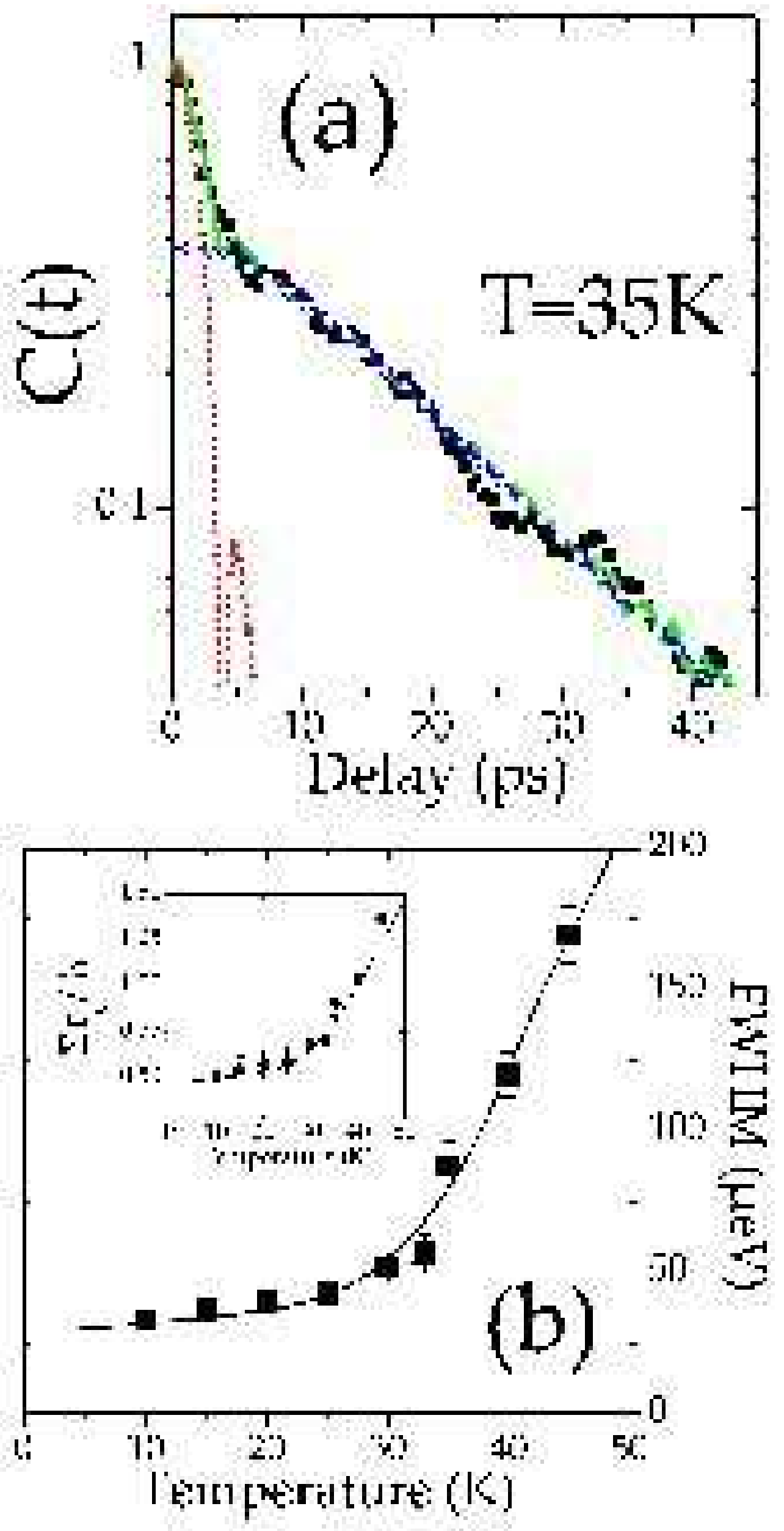